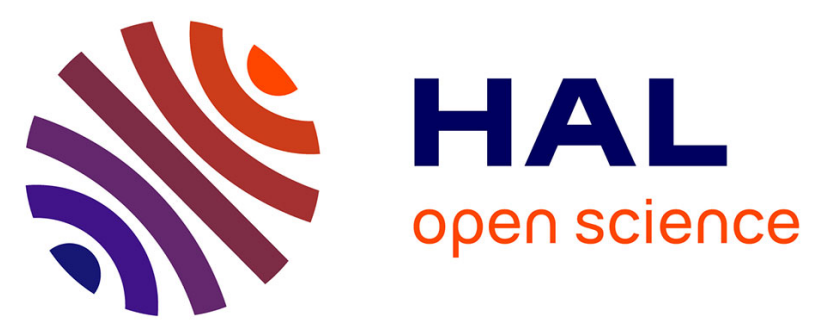

\title{
Squeeze film damping and stiffening in circular CMUT with air-filled cavity: Influence of the lateral venting boundary conditions and the bias voltage
}

\author{
Ayrat Galisultanov, Patrice Moal, Gilles Bourbon, Vincent Walter
}

\section{- To cite this version:}

Ayrat Galisultanov, Patrice Moal, Gilles Bourbon, Vincent Walter. Squeeze film damping and stiffening in circular CMUT with air-filled cavity: Influence of the lateral venting boundary conditions and the bias voltage. Sensors and Actuators A: Physical , 2017, 266, pp.15-23. 10.1016/j.sna.2017.09.003 . hal-02131278

\author{
HAL Id: hal-02131278 \\ https://hal.science/hal-02131278
}

Submitted on 23 Jul 2019

HAL is a multi-disciplinary open access archive for the deposit and dissemination of scientific research documents, whether they are published or not. The documents may come from teaching and research institutions in France or abroad, or from public or private research centers.
L'archive ouverte pluridisciplinaire HAL, est destinée au dépôt et à la diffusion de documents scientifiques de niveau recherche, publiés ou non, émanant des établissements d'enseignement et de recherche français ou étrangers, des laboratoires publics ou privés. 


\section{Squeeze Film Damping and Stiffening in Circular CMUT with Air-Filled Cavity: Influence of the lateral venting boundary conditions and the bias voltage}

The present paper deals with the analysis of the squeeze film effects in circular capacitive micromachined ultrasound transducers (CMUT) operating in air, with emphasis on improved bandwidth. Firstly, a 1D analytical approach based on parallel plate approximation is recalled. The opposing influences of the electrostatic softening and the squeeze film stiffening make the resonant frequency dependent on the bias voltage with respect to air spring constant to mechanical spring constant ratio. In a second part, FEM models using COMSOL Multiphysics ${ }^{\circledR}$ are built to analyze the influences of the plate flexibility and the lateral venting boundary conditions on the squeeze film effects. The associated numerical results show that viscous losses are involved in sealed air-filled cavities. Moreover, the dimensionless elastic and viscous damping forces do not depend on the lateral venting boundary conditions for high squeeze number range, usual for CMUT operation. Finally, 2D full coupled simulations of flexible CMUT are compared with experimental data. Thus, the squeeze film damping increases bandwidth of aircoupled CMUTs with both sealed and laterally vented cavities.

Keywords: squeeze film damping; vented CMUT; sealed CMUT; bias voltage; resonant frequency; bandwidth.

\section{Introduction}

Typical capacitive micromachined ultrasound transducers (CMUT) consist of a metallized membrane suspended over an evacuated or air-filled cavity, the height of which corresponds to main contribution to the electrostatic gap (Fig. 1). When the top of the membrane is loaded by a fluid or a gas, the membrane vibration can be used for ultrasound waves transmitting or receiving in immersion [1-3] and air-coupled [4-9] applications.

Air-coupled sensing applications like flow metering [10], ultrasound imaging [11] and acoustic emission detection in solids [12] require high values of two key figures of merit, the sensitivity and the bandwidth. The sensitivity of CMUTs is mainly related to the DC bias voltage decreasing the electrostatic gap and thus increasing the capacitance variation [13]. The bandwidth of air-coupled CMUTs with evacuated cavity is very narrow. It is limited by the low acoustic impedance of air. Several approaches were proposed in the literature for bandwidth extension. One can fabricate a device with membranes of different radii [14] or use selective networks to electronically manipulate the resonant behavior [15].

The most promising way for bandwidth extension is to use CMUTs with air-filled cavities $[4,5,8]$. The bandwidth of such CMUT increases due to squeeze film effects in the cavity and the resistance to the air flow through the venting holes. In order to study the effects of the squeeze film only, non-perforated circular CMUTs with lateral channels for the bottom electrode connection are fabricated using a wafer bonding technology process [16]. Within this context, the present work aims at providing a contribution to the analysis of the squeeze film effects in circular CMUTs by considering the influence of the DC bias voltage and the real lateral venting boundary conditions at the periphery of the CMUT cavity; i.e. quasi-sealed conditions for CMUTs only vented by narrow channels. The squeeze film effects are driven by both stiffening and damping mechanisms.

The stiffening effect of the air film in the back cavity increases the resonant frequency of a vented [4,5] and sealed [17] CMUT. The pure spring force assumption [17,18] allows us to consider the squeeze film effect simply as an additional stiffness of uniform air gap (Fig. 1.a) in the existing lumped parameter models of CMUT $[9,19,20]$. However, the DC bias voltage 
causes the static deflection of the membrane, making the cavity non-uniform. The models based on finite difference plate equation discretization take into account this effect [21-25]. Therefore, the air stiffness of non-uniform circular cavity (Fig. 1.b) have to be determined to extend the scope of the elastic spring force assumption.

The damping mechanisms have been investigated for a gas film trapped between two movable parallel plates. The behavior of the gas film can be defined as a solution of the Reynolds equation for compressible gas [26]. In this case, the viscous damping force occurs only for open vented lateral boundary conditions (BC), and is induced by the gas flow in and out of the cavity [17,18]. However, such models are not consistent with the behavior of elastically deformable CMUTs membranes. Furthermore, the models taking into account a flexible membrane lead to contradictory conclusions. In [27], the flexibility of the vibrating plate has been demonstrated to cause viscous damping for a sealed structure. On the other hand, Ahmad et al. [28] have showed that the resulting air flow inside a completely sealed circular CMUT cavity produces an air spring force with no dissipation. In order to clarify this contradiction, elastic and damping forces due to the squeeze films effects have to be assessed according to realistic geometry and boundary conditions.

In the first part of this paper, a simple 1D analytical approach allows to describe the evolution of the resonant frequency as a function of the DC bias voltage related to the stiffness ratio between the air film and the piston. The combination of air stiffening effect in the back cavity and the electrostatic softening effect lead to opposing influences on the resonant frequency. Regarding the influence of the DC bias voltage on the geometry cavity, an "effective" gap constant is introduced as a parameter which allows to define the air stiffness of a non-uniform circular cavity as a function of the maximum displacement of the plate.

In a second part, we propose a numerical approach based on the FEM Multiphysics $\mathrm{COMSOL}^{\circledR}$ software to define the dimensionless elastic and viscous damping forces in circular CMUT with flexible plate. For high squeeze number, typical for CMUT operation, air spring constants for piston and flexible membrane are similar. However, a viscous damping force for flexible membranes is present for both sealed and vented lateral boundary conditions (BC) and does not depend on venting BC. This loss is caused by air motion inside the cavity. Thus, the viscous damping characteristic of such CMUTs provides the possibility to increase the bandwidth for air-coupled applications. Finally, the experimental results are compared with the results of analytical calculation of resonant frequency as well as with the results of FEM simulations of resonant frequency and bandwidth. Both calculation approaches show a good agreement with experimental data.

\section{1D electromechanical piston-like model of a circular CMUT with air-filled cavity}

In this section, the CMUT is considered as a rigid circular piston of radius $a$, thickness $t$ and relative permittivity $\varepsilon_{t}$. The piston is suspended above an air-filled cavity with the same radius and a thickness $g_{0}$ (Fig.1.a). The top of the piston is metallized, but the electrode thickness is neglected in the following. The bottom electrode is covered by an insulation layer 
of thickness $i$ and relative permittivity $\varepsilon_{i}$. The mass $m$ and the spring constant of the piston $k_{S}$ are equivalent parameters representing respectively the mass and the stiffness of the CMUT membrane. The mass of the piston $m$ equals $\rho t S$, where $\rho$ is the piston's material density, and $S=\pi a^{2}$ is the area of the circular piston.

\subsection{Electromechanical behavior of the piston-like CMUT}

A 1D parallel plate transducer model for evacuated CMUT is developed successfully in [13]. When the DC bias voltage $V_{D C}$ is applied between the top and the bottom electrode, the electrostatic attraction is:

$$
F_{e}=\frac{\varepsilon_{0} S V_{D C}^{2}}{2\left(d_{e f f}-x\right)^{2}}
$$

where $\varepsilon_{0}$ is the permittivity constant of vacuum and $d_{e f f}=g_{0}+t / \varepsilon_{t}+i / \varepsilon_{i}$ is the effective electrostatic gap distance. This force causes a static displacement $x$ of the piston that is defined by the following equilibrium equation related to the restoring force of the piston equivalent spring $k_{s}$ :

$$
\frac{\varepsilon_{0} S V_{D C}^{2}}{2\left(d_{e f f}-x\right)^{2}}=k_{S} x
$$

The displacement at the collapse point is one-third of the effective electrostatic gap and the collapse voltage is given by:

$$
V_{c o l}=\sqrt{\frac{8 k_{\mathrm{s}} d_{e f f}^{3}}{27 S \varepsilon_{0}}} .
$$

\subsection{Squeeze film elastic and viscous damping forces acting on a piston-like CMUT}

The superposition of a DC voltage $V_{D C}$ and a small signal $V_{A C}$ voltage applied between the CMUT electrodes leads to the piston vibration at the frequency of the AC excitation. The behavior of a trapped gas film between two parallel vibrating plates could be found as a solution of the linearized Reynolds equation for compressible gas [18]. This equation neglects the fluid inertia and uses the isothermal condition. A solution for vented circular plates in parallel motion is given by Crandall [29]. He uses simplified boundary conditions and vanished acoustical pressure at the edges of the moving plates. The dimensionless viscous $f_{d}(\sigma)$ and elastic $f_{e}(\sigma)$ damping forces are respectively:

$$
\begin{gathered}
f_{d}(\sigma)=\left(\sqrt{\frac{2}{\sigma}} \frac{b e r \sqrt{\sigma}\left(\operatorname{ber}_{1} \sqrt{\sigma}+b e i_{1} \sqrt{\sigma}\right)+b e i \sqrt{\sigma}\left(b e i_{1} \sqrt{\sigma}-b e r_{1} \sqrt{\sigma}\right)}{(b e r \sqrt{\sigma})^{2}+(b e i \sqrt{\sigma})^{2}}\right) \\
f_{e}(\sigma)=\left(1-\sqrt{\frac{2}{\sigma}} \frac{b e r \sqrt{\sigma}\left(b e i_{1} \sqrt{\sigma}-b e r_{1} \sqrt{\sigma}\right)-b e i \sqrt{\sigma}\left(b e r_{1} \sqrt{\sigma}+b e i_{1} \sqrt{\sigma}\right)}{(b e r \sqrt{\sigma})^{2}+(b e i \sqrt{\sigma})^{2}}\right), \\
\sigma=\frac{12 \mu \omega a^{2}}{P_{a} g_{0}^{2}}
\end{gathered}
$$

where $\sigma$ is called the squeeze number (6), $\mu$ is the dynamic viscosity of air, $\omega$ is the angular frequency of the vibration, $P_{a}$ is the ambient pressure, ber, ber ${ }_{1}$, bei and $b e i_{1}$ are the KelvinBessel functions of order 0 and 1 . 
In the case of low frequency oscillation and consequently low squeeze number, the plate moves slowly and the gas has time to "leak" out from the gap. In this case, the viscous damping force dominates. At the opposite, for high frequency oscillation, the gas film is mainly compressed and has no time to "leak" out. In this case, the elastic force dominates and the air film acts as a spring [18].

For sealed cavity and parallel plate motion, Darling et al. [30] demonstrated a pure spring force with no viscous damping for vibrating parallel rectangular plates. In this approach, we assume the same behavior for the sealed circular plate, i.e. $f_{d}(\sigma)=0$ and $f_{e}(\sigma)=1$. The comparison of solutions for vented and sealed air-filled cavities is presented in Fig. 2. The coefficient of viscous damping force $c_{d}$ and the air spring constant $k_{e}$ could be found as:

$$
\begin{aligned}
& c_{d}=\frac{P_{a} S}{\omega g_{0}} f_{d}(\sigma), \\
& k_{e}=\frac{P_{a} S}{g_{0}} f_{e}(\sigma) .
\end{aligned}
$$

In CMUTs, the typical distance $g_{0}$ between the electrodes is in the micrometer range or lower. Thus, the mean free path length of the air $\lambda=65 \mathrm{~nm}$ [31] becomes not negligible compared with the air-filled gap size. Veijola et al. [32] presented a simple approximate equation which takes into account the rarefaction effect via an effective viscosity defined as:

$$
\mu_{e f f}=\frac{\mu}{1+9.638 \cdot K n^{1.159}},
$$

where $K n=\lambda / g_{0}$ is the Knudsen number. For $K n<0.01$ the Navier-Stokes and Reynolds continuum equations could be used without any modifications. If $0.01<K n<0.1$, the viscosity constant in the squeeze number expression (6) needs to be replaced by relation (9).

\subsection{Electrostatic softening against squeeze film stiffening}

The dynamic behavior of the parallel plate circular transducer with an air-filled cavity is described by the following differential equation of motion:

$$
m \ddot{x}+c_{d} \dot{x}+\left(k_{s}+k_{e}\right) x=F_{0} \sin (\omega t),
$$

where $F_{0}$ is the amplitude of the small signal harmonic excitation. In this equation, we assume that the movable piston is not loaded by any medium at the top.

For standard CMUTs dimensions and operating frequencies, the squeeze number is high and the pure air-film spring assumption for the CMUT with vented cavity can be considered: $k_{e} \cong P_{a} S / g_{0}, c_{d} \cong 0[17,18]$. With this assumption, the air spring constant is a function of the gap size, and, consequently, the DC bias voltage level. Applied DC bias voltage causes both the stiffening effect of the squeeze air film in the back cavity and the electrostatic softening effect. The total stiffness $k$ as a function of the membrane displacement $x$ for CMUT with vented cavity becomes:

$$
k=k_{s}\left(1-\frac{2 x}{d_{e f f}-x}\right)+k_{e}\left(1+\frac{x}{g_{0}-x}\right) .
$$

For relatively thin insulation and membrane layers $g_{0} \gg t / \varepsilon_{t}+i / \varepsilon_{i}$ and equation (11) can be simplified as: 


$$
k=k_{s}+k_{e}+\left(k_{e}-2 k_{s}\right) \frac{x}{g_{0}-x} .
$$

Eq. (11)-(12) could be also used for the case of sealed CMUT considering a stable pressure assumption inside the cavity.

The natural resonant frequency $\omega_{n}$ and bandwidth $\Delta \omega$ for both open-vented (high squeeze number) and sealed boundary conditions are given respectively by:

$$
\begin{aligned}
& \omega_{n}=\sqrt{\frac{k}{m}}, \\
& \Delta \omega=\frac{c_{d}}{m} \cong 0 .
\end{aligned}
$$

The dependence of the normalized frequency $\omega_{n} / \omega_{0}$ on the normalized DC bias voltage

$V_{D C} / V_{c o l}$ for different $k_{e} / k_{s}$ ratio is presented in Fig. 3, where $\omega_{0}=\sqrt{k_{s} / m}$ is the pure mechanical eigenfrequency with an evacuated cavity. The curves are calculated from Eq. (2) and (12). The analysis of results shows that for $k_{e} / k_{s}=2$ the natural eigenfrequency of piston vibration does not depend on applied DC bias voltage and equals to $\sqrt{3} \omega_{0}$. The resonant frequency of CMUT cell with air-filled cavity in the vicinity of collapse point is mainly defined by the air spring constant and tends to $\sqrt{3 k_{e} /(2 m)}$.

Experimental characterizations of the resonant frequency are performed on a $167 \mu \mathrm{m}$ radius plate at three pressure levels in a rough vacuum chamber: $50 \mathrm{~Pa}, 18.5 \mathrm{kPa}, 31.5 \mathrm{kPa}$ and at the atmospheric pressure, i.e. around $101 \mathrm{kPa}$. The corresponding ratios $k e / k s$ are respectively about $0(50 \mathrm{~Pa}), 1.2(18.5 \mathrm{kPa}), 2.0(31.5 \mathrm{kPa})$ and $6.4(101 \mathrm{kPa})$. The Fig. 4 shows a reasonably good correlation between the experimental data and the 1D model predictions. The influence of the squeeze film damping on the resonant frequency at low DC bias voltage (increasing of the resonant frequency with the surrounding pressure) and the competition between the electrostatic softening and the squeeze film stiffening (depending on whether the ratio ke/ks is higher or lower than approximately 2) are properly estimated validating the interest of the 1D model at the pre-sizing phase.

\section{Squeeze film effects from 2D FEM simulations in the mechanics-thermoacoustics domains}

In this part, the CMUT is considered in a more realistic way as a clamped circular flexible plate. In the following, 2D FEM simulations from COMSOL Multiphysics ${ }^{\circledR}$ software allows to discriminate some trends about the influence of the plate flexibility on the squeeze film effect. Further we define air spring constant of non-uniform cavity caused by a DC bias voltage, by a pressure difference or by a stress release. An "effective" gap constant is introduced as parameter which allows to define the air spring constant as function of the maximum displacement of the clamped circular plate.

3.1. Elastic and viscous damping forces under open-vented and sealed boundary conditions 
A 2D axisymmetric model of the circular plate over a uniform thin air gap is firstly implemented to discriminate the influence of the plate flexibility. No surrounding medium is taken into account above the top of the plate to focus on squeeze film effects. Navier-Stokes equations within the COMSOL Thermoacoustics module are used to model the squeeze film effects in the air-filled cavity. Both open-vented and sealed boundary conditions are considered in the simulations in order to clarify their respective impact. For both cases, the pressure in cavity $P_{a}$ is assumed to be stable and equals to one atmosphere $(101.3 \mathrm{kPa})$. We assume no pressure difference between cavity and ambient environment. The membrane is made of silicon and is $2.3 \mu \mathrm{m}$ thick. The air-filled gap thickness $\left(g_{0}\right)$ is varied from $1 \mu \mathrm{m}$ to $40 \mu \mathrm{m}$, and the rarefaction effect is ignored. Table 1 reports the material properties of silicon (isotropic assumption with averaged values) and aluminum. The excitation signal is a uniform pressure applied to the top of the plate and the displacement response of the system is calculated according to a harmonic analysis.

Table 1. Material parameters of Silicon and Aluminum

\begin{tabular}{cccc}
\hline \multicolumn{4}{c}{ Mechanical material properties } \\
\hline & $\begin{array}{c}\text { Young's modulus } \\
(\mathrm{GPa})\end{array}$ & Poisson's ratio & $\begin{array}{c}\text { Density } \\
\left(\mathrm{kg} / \mathrm{m}^{3}\right)\end{array}$ \\
\hline $\mathrm{Si}(100)$ & 148.8 & 0.176 & 2329 \\
\hline $\mathrm{Al}$ & 70 & 0.35 & 2700 \\
\hline
\end{tabular}

Without any DC bias voltage, the total system stiffness is a sum of mechanical and air stiffness, i.e. $k=k_{s}+k_{e}$. In other words, the difference between the resonant frequency of the flexible plate vibrating over a uniform thin air gap $\omega_{d}$ and the resonant frequency of the same plate in vacuum $\omega_{0}$ is defined by the air-spring constant. By neglecting the difference between damped and natural resonant frequency, the air-spring constant can be calculated as:

$$
k_{e}=m\left(\omega_{d}^{2}-\omega_{0}^{2}\right),
$$

where $m$ is the equivalent mass of the plate which is assumed to be the real mass of the silicon membrane. This assumption will be discussed in the following section. Thus, the dimensionless elastic damping force is expressed from Eq. (8) and (15) and equals:

$$
f_{e}(\sigma)=\frac{m\left(\omega_{d}^{2}-\omega_{0}^{2}\right)}{P_{a} S / g_{0}}
$$

As the top of the moving membrane is not loaded by any fluid or gas, the bandwidth of this system is completely determined by the squeeze film damping. Thus, the dimensionless viscous damping force $f_{d}(\sigma)$ can be defined from Eq. (7) and (15) as:

$$
f_{d}(\sigma)=\frac{\Delta \omega}{\omega_{d}} \cdot \frac{m \omega_{d}^{2}}{P_{a} S / g_{0}}
$$

The extraction of the dimensionless elastic and viscous damping force constants according to equations (16) and (17) is performed. Fig. 5 shows the results for a $150 \mu \mathrm{m}$ radius plate and both open-vented and sealed boundary conditions. Fig. 6 represents the results for $50 \mu \mathrm{m}, 75 \mu \mathrm{m}$ and $150 \mu \mathrm{m}$ radius plates and sealed boundary conditions.

\subsection{Discussion and significant outcomes}


For high squeeze numbers and any radius and boundary condition (cf. Fig. 5 and 6), the dimensionless elastic damping force tends to 1 . Consequently, the air-spring constant is equal to: $k_{e}=P_{a} S / g_{0}$. Thus, the air-spring constant of a given flexible plate is equivalent to that of a piston with the same mass and radius. Additionally, this justifies the choice of the real mass used to identify the dimensionless elastic and viscous damping forces in equations (16) and (17). For low squeeze number, the numerical simulations do not predict accurately the dimensionless elastic and viscous damping forces by equations (16) and (17). This is highlighted by a shaded area in the low squeeze number region in Fig. 5 and 6. It will be noted that the dimensionless elastic and viscous damping forces are derived from the solution of linearized Reynolds equation neglecting fluid/gas inertia. However, the numerical simulations based on the Navier-Stokes equations solution take into account the inertia effects of air. For low squeeze number, air-spring constant decreases dramatically due to the gap increase. On the other hand, the added mass of air becomes not negligible and decreases the resonant frequency of the plate. Thus, it contradicts our assumption that the difference of the resonant frequencies is mainly governed by the air-spring constant.

The comparison of the results for the dimensionless viscous damping force clearly shows the difference between the fluid/gas behavior under a flexible plate and a piston for both open-vented and sealed boundary conditions. For the piston parallel motion, the pressure difference between the squeeze air film and the boundary of open cavity causes the air to flow inside and outside the cavity. Obviously, this air motion induces viscous losses. In the case of sealed cavity, there is no pressure difference, no air motion and, consequently, no viscous loss (Fig. 2). For the flexible plate, due to the non-uniform profile of the vibration velocity, the pressure is non-uniform and it reaches its maximum at the center of the cavity. This pressure difference causes an air flow inside the sealed cavity, and, consequently, viscous loss. For high squeeze numbers, typical for CMUT operation, the viscous damping force is similar for both open-vented and sealed boundary conditions (cf. Fig. 5). This can be related to the lateral velocity profile, that is the same inside the cavity for both type of vented boundary conditions (cf. Fig. 7.a). The velocity profile along the center of open and sealed cavity for a $150 \mu \mathrm{m}$ radius membrane is identified in the vicinity of the resonant frequency for two gap size $1 \mu \mathrm{m}$ and $10 \mu \mathrm{m}$, corresponding to two squeeze number values, 239 and 1.3 respectively. The velocity profile in Fig. 7.a demonstrates that the gas in cavity with open boundary has no time to "leak" from external border. This shows that the velocity profile is not sensitive to the type of vented boundary condition. When the gap increases or when the squeeze number decreases, the air flow in and out of open cavity rises (Fig. 7.b). Thus, the results of FEM simulations demonstrate that flexible plate and piston under squeeze film effect have completely different loss behaviors.

Additionally, it can be noticed that the dimensionless elastic and viscous damping forces are not stable with the plate radius for a given boundary condition (cf. Fig. 6). However, as mentioned above, their respective values are similar for high squeeze number with the following trends: $f_{e}(\sigma) \sim 1$ and $f_{d}(\sigma) \sim \sigma^{-0.89}$.

Because typical CMUT operations involve squeeze number usually higher than 50 , the following outcomes can be deduced:

- The dimensionless elastic damping force can be considered close to 1 , 
- The dimensionless elastic and viscous damping forces do not depend on the lateral vented boundary conditions.

In further bandwidth and frequency spectrum calculations, the last outcome allows to implement a 2D axisymmetric model. It is less time-consuming than a 3D CMUT model with non-axisymmetric vented conditions like lateral vented channels.

\subsection{Influence of DC voltage: introduction of an "effective" gap}

The previous developments allow us to determine the dimensionless elastic and viscous damping forces in the case of uniform air gap. The CMUT operation usually requires a high DC bias voltage, which creates a non-uniform air gap (Fig. 1.b). Therefore, an accurate implementation of the squeeze film effects in existing analytical and numerical CMUTs models as described in [21-25, 33] requires the characterization of the stiffness of non-uniform airfilled gap. The problem is handled only for large squeeze number, typical for CMUT operation. The non-uniform air gap is directly introduced at the geometrical level, i.e. only the change of the air stiffness affects the resonant frequency.

The gap non-uniformity is considered according to a deflection profile corresponding to a uniform pressure loading as [33]:

$$
g(r)=g_{0}-w_{\max }\left(1-\left(\frac{r}{a}\right)^{2}\right)^{2}
$$

with $-g_{0} / 2 \leq w_{\max } \leq g_{0} / 2$. It can be noticed that both sign of $w_{\max }$ are investigated to allow initial deflection from different causes to be addressed. The initial air gap thickness is $g_{0}=$ $0.75 \mu \mathrm{m}$, and two plate radius are simulated: $50 \mu \mathrm{m}$ and $150 \mu \mathrm{m}$. The rarefaction effect is not taken into account.

From previous calculation, the air-filled gap is supposed to operate in the elastic regime. In this case, it is assumed that the air film stays in the same regime after the introduction of the gap non-uniformity by eq. (18). The non-uniform gap changes the air-spring constant, which can be calculated from (15). A uniform "effective" gap $g_{\text {eff }}$ with the same air spring constant is then introduced as:

$$
g_{e f f}=g_{0}+\gamma w_{\max },
$$

where $\gamma$ is the "effective" gap constant. The value of this constant is calculated from equation (20) for the above mentioned range of maximal deflection values according to the FEM identification of the resonant frequency.

$$
\gamma=\frac{g_{0}}{w_{\max }}\left[\frac{P_{a} S / g_{0}}{m\left(\omega_{d}^{2}-\omega_{0}^{2}\right)}-1\right]
$$

The results reported on Fig. 8 show an average value of the "effective" gap constant for both radius around 0.73 . Once the "effective" gap constant is calculated, we can determine the non-uniform air-filled cavity stiffness as a function of the maximum plate displacement from eq. (21):

$$
k_{e}=\frac{P_{a} S}{g_{0}+0.73 w_{\max }}
$$


Thus, the knowledge of the "effective" gap constant and the maximal center deflection enables us to define the air spring constant. Fig. 9 shows the air spring constant based results and experimental data about the evolution of the resonant frequency with respect to the DC bias voltage for $67 \mu \mathrm{m}$ and $167 \mu \mathrm{m}$ radius plates. This acceptable comparison supports the air spring constant assumption of non-uniform gap to reflect the squeeze film stiffening in different modeling strategies.

\section{2D full coupled FEM simulations of a flexible plate CMUT with air-filled cavity}

2D FEM simulations in the mechanics-thermoacoustics domains illustrate the nondependence of the squeeze film effects to the vented boundary conditions. This justifies the validity of 2D axisymmetric models to model actual 3D architecture of CMUTs, especially related to non-axisymmetric vented boundary conditions. Therefore, in this part, 2D full coupled FEM simulations of CMUT including mechanics, electrostatics, and thermoacoustics domains are performed and compared to experimental data.

\subsection{Experimental characterization}

In previous work [16], a fabrication process of CMUTs based on the anodic bonding of a SOI wafer on a borosilicate glass wafer with lateral channels for the bottom electrode connection has been presented. In this process, the SOI device layer defines the membrane thickness. In the present work, single CMUT cells are fabricated with four different radii: $67 \mu \mathrm{m}$, $87 \mu \mathrm{m}, 117 \mu \mathrm{m}$ and $167 \mu \mathrm{m}$. The silicon plate thickness and the resistivity are respectively $2.3 \mu \mathrm{m}$ and $1-30 \Omega \cdot \mathrm{cm}$. The aluminum top electrode covering the silicon membrane and the gold/chromium bottom electrode are $0.22 \mu \mathrm{m}$ and $0.25 \mu \mathrm{m}$ thick, respectively. The initial airfilled gap between the silicon plate and the bottom electrode is equal to $0.75 \mu \mathrm{m}$. Table $2 \mathrm{a}$ summarizes the main experimental results in terms of static deflection, collapse voltage, resonant frequency of the first bending mode and the corresponding bandwidth. For comparison, expected resonant frequencies from the 1D model (cf. § 2.3) are reported in Table $2 \mathrm{~b}$ in the case of experimental ambient pressure configurations reported in Table $2 \mathrm{a}$ (i.e., $1 \mathrm{~atm}$ or $101 \mathrm{kPa}$ and $50 \mathrm{~Pa}$ ). Besides, the simple and fast 1D model is run in order to simulate intermediate ambient pressure configurations and illustrate the influence of environment on the resonant frequencies.

Table 2a. Experimental results

\begin{tabular}{ccccccc}
\hline $\begin{array}{c}\text { Radius } \\
(\mu \mathrm{m})\end{array}$ & $\begin{array}{c}\text { Bottom } \\
\text { electrode } \\
\text { radius }(\mu \mathrm{m})\end{array}$ & $\begin{array}{c}\text { Static } \\
\text { deflection } \\
(\mathrm{nm})\end{array}$ & $\begin{array}{c}\text { Pull-In } \\
\text { voltage }(\mathrm{V})\end{array}$ & $\begin{array}{c}\text { Frequency } \\
(\mathrm{kHz}), \\
\mathrm{P}_{\mathrm{a}}=1 \mathrm{~atm}\end{array}$ & $\begin{array}{c}\text { Frequency } \\
(\mathrm{kHz}), \\
\mathrm{P}_{\mathrm{a}}=50 \mathrm{~Pa}\end{array}$ & $\begin{array}{c}\text { Bandwidth } \\
(\mathrm{kHz}), \\
\mathrm{P}_{\mathrm{a}}=1 \mathrm{~atm}\end{array}$ \\
\hline 67 & 40 & 80 & 132 & 2125 & 2012 & 36.5 \\
\hline 87 & 56 & 160 & 83 & 1375 & 1154 & 43 \\
\hline 117 & 80 & 277 & 34 & 982 & 657 & 44.4 \\
\hline 167 & 120 & 80 & 16 & 824 & 326 & 40.6 \\
\hline
\end{tabular}


Table 2b. Expected resonant frequencies from the 1D model (cf. § 2.3)

\begin{tabular}{ccccccc}
\hline $\begin{array}{c}\text { Radius } \\
(\mu \mathrm{m})\end{array}$ & $\begin{array}{c}\text { Frequency } \\
(\mathrm{kHz}),\end{array}$ & $\begin{array}{c}\text { Frequency } \\
(\mathrm{kHz}),\end{array}$ & $\begin{array}{c}\text { Frequency } \\
(\mathrm{kHz}),\end{array}$ & $\begin{array}{c}\text { Frequency } \\
(\mathrm{kHz}),\end{array}$ & $\begin{array}{c}\text { Frequency } \\
(\mathrm{kHz}),\end{array}$ & $\begin{array}{c}\text { Frequency } \\
(\mathrm{kHz}),\end{array}$ \\
\hline 67 & $2101 \mathrm{kPa}$ & $\mathrm{P}_{\mathrm{a}}=80 \mathrm{kPa}$ & $\mathrm{P}_{\mathrm{a}}=60 \mathrm{kPa}$ & $\mathrm{P}_{\mathrm{a}}=40 \mathrm{kPa}$ & $\mathrm{P}_{\mathrm{a}}=20 \mathrm{kPa}$ & $\mathrm{P}_{\mathrm{a}}=50 \mathrm{~Pa}$ \\
\hline 87 & 1406 & 1359 & 2047 & 2016 & 1985 & 1953 \\
\hline 117 & 1023 & 956 & 888 & 814 & 732 & 641 \\
\hline 167 & 858 & 777 & 691 & 592 & 474 & 315 \\
\hline
\end{tabular}

About the Table 2a, the static deflection represents the center deflection of the membrane measured after the fabrication process. It results from the relaxation of the residual stress in the Si device layer. This residual stress may be induced by three main sources: the fabrication process of the SOI wafer, the bonding process, which occurs at a temperature of $400^{\circ} \mathrm{C}$ or the aluminum deposition step on the top of the plate. The quite low values of the static deflection reported in table 2 indicate that the silicon plates are practically free from residual stresses.

The displacement response is measured using a Polytec laser vibrometer with a vibrometer sensor head OFV-534 and a OFV-5000 controller. The resonance frequency and the corresponding bandwidth in the ambient air are collected as a function of the bias voltage. The values at a low DC bias voltage $\left(\mathrm{V}_{\mathrm{DC}}=1 \mathrm{~V}\right)$ are reported in table 2 and all the results are plotted in Fig. 10.

Fig. 10 shows that the evolution of the resonance frequency as a function of the DC bias voltage is strongly dependent on the membrane radius. It decreases for the radii $67 \mu \mathrm{m}$ and $87 \mu \mathrm{m}$. It is constant for the radius $117 \mu \mathrm{m}$ and it increases for the radius $167 \mu \mathrm{m}$. This perfectly illustrates the competition between the electrostatic softening and the squeeze film stiffening (cf. $\$ 2.3$ and equation (12)). As the air-filled gap is constant for all the radius configuration, the squeeze film stiffening becomes dominant for the larger plate radius. In other words, from lower to higher radii, the configuration shifts from a mechanical spring to an air-spring dominated field. The ratio between the ambient air (experimental) and vacuum resonant (theoretical) frequencies for the configuration showing no influence of the DC bias voltage on the resonance frequency $(117 \mu \mathrm{m}$ radius) is around 1.5 , a value quite close to $\sqrt{3}$ given by the simple equivalent analytical approach (cf. §2.3).

The corresponding bandwidths as a function of the DC bias voltage globally show opposite trends, i.e.: an increasing in the case of the mechanical spring dominated area $(67 \mu \mathrm{m}$

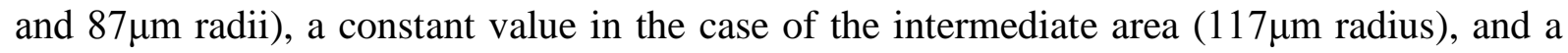
slight decreasing in the case of the air-spring dominated area $(167 \mu \mathrm{m}$ radius). For the $67 \mu \mathrm{m}$ radius CMUT, it can be noticed that the collected bandwidth as a function of the DC bias voltage raises from $36.5 \mathrm{kHz}\left(\mathrm{V}_{\mathrm{DC}}=1 \mathrm{~V}\right)$ to $100.2 \mathrm{kHz}\left(\mathrm{V}_{\mathrm{DC}}=125 \mathrm{~V} \approx 0.95 \mathrm{~V}_{\mathrm{COL}}\right)$. This results demonstrate the relevance of designing CMUT with sealed air-filled cavities for air-coupled applications. Besides, the DC bias voltage variation provides a way to control the bandwidth.

\subsection{D full coupled FEM simulations and discussion}


In this part, a 2D axisymmetric full coupled model is developed in COMSOL Multiphysics ${ }^{\circledR}$ software combining Thermoacoustics and Electromechanics interfaces. The objective is to calculate the dynamic response of a CMUT cell taking into account the electromechanical behavior in relation with the DC bias voltage, the squeeze film effects occurring in the air-filled back cavity, and the radiation losses in the surrounding medium. As discussed above, the real lateral venting channels are neglected by considering completely sealed cavities. This assumption is validated according to the squeeze number range from 230 to 545 for the four radii tested configurations.

The radiation losses in the surrounding air are introduced by considering the air loading on the top of the plate. The perfectly matched layer (PML) is added to simulate an open and non-reflecting infinite domain. For the accurate radiation loss calculation, we used at least 10 elements per wavelength.

The combined COMSOL Thermoacoustics and Electromechanics interfaces are implemented using two succesive steps: a stationary study and a perturbation study in the frequency domain. The stationary study provides the static deflection of the plate due to the residual stress (chosen according to the static deflection) and especially to the DC bias voltage. In the second step (Frequency Domain, Perturbation Study), a small electrostatic excitation signal is applied and the respective resonant frequency and bandwidth are obtained.

The geometric and physical parameters of the circular plate are the same than in the fabricated devices mentioned above. The viscosity constant is replaced by eq. (9), in which the initial gap size of $0.75 \mu \mathrm{m}$ is used. The silicon membrane has a relatively high conductivity (1$30 \Omega \cdot \mathrm{cm}$ ), therefore the effective top electrode is located at the bottom side of the plate.

The FEM calculated evolutions of the resonance frequency as a function of the bias voltage and the corresponding bandwidth are reported in Fig. 10 for comparison with the experimental data. The FEM simulations accurately fit the pull-in voltage and the resonant frequency as a function of the DC bias voltage. Moreover, the bandwidth for low DC bias voltage is also perfectly predicted. The good correlation between numerical and experimental data validates the proposed approach of replacing the real CMUT architecture with nonaxisymmetric lateral venting channels to axisymmetric sealed cavity geometries.

However, the FEM model does not accurately estimate the corresponding bandwidth for high bias voltage level, especially for the lowest value of the radius $(67 \mu \mathrm{m})$. It is assumed that this discrepancy is connected to an incorrect accounting of the effective viscosity. Firstly, Veijola et al. [32] approximation is used for a parallel plate motion with a uniform gap, which is not adapted to the deflected plate for a high DC bias voltage. Finally, the gap reduction for a high DC bias voltage leads to a non-continuous regime of the fluid flow as the Knudsen number becomes higher than 0.1 . For this regime, the Navier-Stokes equations become less appropriate to describe the behavior of the gas. A specific analysis of the fluid flow in the molecular regime would be required to describe such a rarefied fluid flow should, for example by implementing the Molecular flow module or Microfluidics module of the COMSOL ${ }^{\circledR}$ package. 


\section{Conclusion}

The present work focuses on the characterization of the squeeze film effects in CMUTs with air-filled cavities and more particularly on the effects of the DC bias voltage. First, a 1D analytical equivalent approach, based on a rigid piston model, demonstrates the competition between the electrostatic softening related to the DC bias voltage and the squeeze film stiffening due to gap reduction of the air-filled back cavity. Mechanical spring and air-spring dominated areas are highlighted and a stiffness ratio between air spring and mechanical spring involves a resonant frequency stability with regard to the DC bias voltage. Furthermore, numerical simulations with the COMSOL Multiphysics ${ }^{\circledR}$ software are undertaken in order to quantify the squeeze film effects in flexible CMUTs for different laterally vented boundary conditions and plate radii. For high squeeze numbers, the dimensionless elastic and viscous damping forces do not depend on the laterally vented boundary conditions. The elastic component of squeeze film in flexible plates for sealed and open-vented boundary conditions is similar to the value found for the sealed piston. However, a viscous damping component in flexible membranes is present due to the non-uniform pressure distribution and air motion inside the air-filled back cavity. Thus, the viscous damping characteristic of sealed or laterally vented CMUTs provides a way to increase the bandwidth for air-coupled applications. Finally, 2D full coupled simulations of flexible CMUT fit accurately the experimental data. As a consequence, the squeeze film effects resulting from the air-filled cavities are effective design parameters that have to be taken into account in the evaluation of the mechanical stiffness of the CMUT membrane. A relevant combination of both mechanical and air springs can be used to make the resonant frequency insensitive to the DC bias voltage. Simultaneously, the DC bias voltage could be considered as a control parameter of the bandwidth in some cases.

\section{Acknowledgment}

This work was supported by the Labex ACTION project (contract "ANR-11-LABX-01-01") and was partly supported by the French RENATECH network and its FEMTO-ST technological facility.

\section{References}

[1] B.T. Khuri-Yakub, O. Oralkan, Capacitive micromachined ultrasonic transducers for medical imaging and therapy, J. Micromech. Microeng. 21 (2011) 54004-54014. doi:10.1088/0960-1317/21/5/054004.

[2] A.S. Savoia, G. Caliano, M. Pappalardo, A CMUT probe for medical ultrasonography: From microfabrication to system integration, in: IEEE Trans. Ultrason. Ferroelectr. Freq. Control, 2012: pp. 1127-1138. doi:10.1109/TUFFC.2012.2303.

[3] B.-G. Jeong, D.-K. Kim, S.-W. Hong, S.-W. Chung, H.-J. Shin, Performance and reliability of new CMUT design with improved efficiency, Sensors Actuators A Phys. 
199 (2013) 325-333. doi:10.1016/j.sna.2013.06.001.

[4] N. Apte, K.K. Park, B.T. Khuri-Yakub, Finite element analysis of CMUTs with pressurized cavities, in: IEEE Int. Ultrason. Symp. IUS, 2012: pp. 979-982. doi:10.1109/ULTSYM.2012.0245.

[5] N. Apte, K.K. Park, A. Nikoozadeh, B.T. Khuri-Yakub, Bandwidth and sensitivity optimization in CMUTs for airborne applications, in: IEEE Int. Ultrason. Symp. IUS, 2014: pp. 166-169. doi:10.1109/ULTSYM.2014.0042.

[6] K.K. Park, H.J. Lee, P. Crisman, M. Kupnik, O. Oralkan, B.T. Khuri-Yakub, Optimum design of circular CMUT membranes for high quality factor in air, in: IEEE Int. Ultrason. Symp. IUS, 2008: pp. 504-507. doi:10.1109/ULTSYM.2008.0123.

[7] H. Nan, K.C. Boyle, N. Apte, M.S. Aliroteh, A. Bhuyan, A. Nikoozadeh, B.T. KhuriYakub, A. Arbabian, Non-contact thermoacoustic detection of embedded targets using airborne-capacitive micromachined ultrasonic transducers, Appl. Phys. Lett. 106 (2015). doi:10.1063/1.4909508.

[8] W.M.D. Wright, S.G. McSweeney, A tethered front-plate electrode CMUT for broadband air-coupled ultrasound, IEEE Int. Ultrason. Symp. IUS. (2013) 1716-1719. doi:10.1109/ULTSYM.2013.0437.

[9] A. Ünlügedik, A. Tas̈delen, A. Atalar, H. Köymen, Designing transmitting CMUT cells for airborne applications, IEEE Trans. Ultrason. Ferroelectr. Freq. Control. 61 (2014) 1899-1910. doi:10.1109/TUFFC.2014.006457.

[10] M.S. Beck, Correlation in instruments: cross correlation flowmeters, J. Phys. E. 14 (2000) 7-19. doi:10.1088/0022-3735/14/1/001.

[11] K.K. Park, B.T. Khuri-Yakub, 3-D airborne ultrasound synthetic aperture imaging based on capacitive micromachined ultrasonic transducers, Ultrasonics. 53 (2013) 1355-1362. doi:10.1016/j.ultras.2013.04.003.

[12] D. Ozevin, D.W. Greve, I.J. Oppenheim, S.P. Pessiki, Resonant capacitive MEMS acoustic emission transducers, Smart Mater. Struct. 15 (2006) 1863-1871. doi:10.1088/0964-1726/15/6/041.

[13] G.G. Yaralioglu, A.S. Ergun, B. Bayram, E. Haeggstrom, B.T. Khuri-Yakub, Calculation and measurement of electromechanical coupling coefficient of capacitive micromachined ultrasonic transducers, Ultrason. Ferroelectr. Freq. Control. IEEE Trans. 50 (2003) 449-456.

[14] M. Kupnik, Min-Chieh Ho, S. Vaithilingam, B.T. Khuri-Yakub, CMUTs for air coupled ultrasound with improved bandwidth, in: 2011 IEEE Int. Ultrason. Symp., IEEE, 2011: pp. 592-595. doi:10.1109/ULTSYM.2011.0143.

[15] S.G. Mc Sweeney, W.M.D. Wright, Improving the bandwidth of air coupled capacitive ultrasonic transducers using selective networks, in: 2008 IEEE Ultrason. Symp., IEEE, 2008: pp. 1191-1194. doi:10.1109/ULTSYM.2008.0287.

[16] M. Bellaredj, G. Bourbon, V. Walter, P. Le Moal, M. Berthillier, Anodic bonding using SOI wafer for fabrication of capacitive micromachined ultrasonic transducers, J. Micromechanics Microengineering. 24 (2014) 25009. doi:10.1088/09601317/24/2/025009.

[17] A. Caronti, G. Caliano, A. Iula, M. Pappalardo, An accurate model for capacitive micromachined ultrasonic transducers, in: IEEE Trans. Ultrason. Ferroelectr. Freq. Control, 2002: pp. 159-168. doi:10.1109/58.985700.

[18] M. Bao, H. Yang, Squeeze film air damping in MEMS, Sensors Actuators, A Phys. 136 (2007) 3-27. doi:10.1016/j.sna.2007.01.008.

[19] H. Kagan Oguz, A. Atalar, H. Köymen, Equivalent circuit-based analysis of CMUT cell dynamics in arrays, IEEE Trans. Ultrason. Ferroelectr. Freq. Control. 60 (2013) 1016-1024. doi:10.1109/TUFFC.2013.2660. 
[20] H. Koymen, A. Atalar, A.S. Tasdelen, Bilateral CMUT Cells and Arrays: Equivalent Circuits, Diffraction Constants and Substrate Impedance, IEEE Trans. Ultrason. Ferroelectr. Freq. Control. (2016) 1-1. doi:10.1109/TUFFC.2016.2628882.

[21] S. Satir, F.L. Degertekin, A nonlinear lumped model for ultrasound systems using CMUT arrays, IEEE Trans. Ultrason. Ferroelectr. Freq. Control. 62 (2015) 1865-1879. doi:10.1109/TUFFC.2015.007145.

[22] B. Shieh, K.G. Sabra, F.L. Degertekin, Efficient broadband simulation of fluidstructure coupling for membrane-type acoustic transducer arrays using the multilevel fast multipole algorithm, IEEE Trans. Ultrason. Ferroelectr. Freq. Control. 63 (2016) 1967-1979. doi:10.1109/TUFFC.2016.2591920.

[23] N. Sénégond, A. Boulmé, C. Plag, F. Teston, D. Certon, Fast time-domain modeling of fluid-coupled cMUT cells: From the single cell to the 1-D linear array element, IEEE Trans. Ultrason. Ferroelectr. Freq. Control. 60 (2013) 1505-1518. doi:10.1109/TUFFC.2013.2723.

[24] D. Certon, F. Teston, F. Patat, A finite difference model for cMUT devices., IEEE Trans. Ultrason. Ferroelectr. Freq. Control. 52 (2005) 2199-2210. doi:10.1109/TUFFC.2005.1563263.

[25] C. Meynier, F. Teston, D. Certon, A multiscale model for array of capacitive micromachined ultrasonic transducers, J. Acoust. Soc. Am. 128 (2010) 2549-2561. doi:10.1121/1.3493433.

[26] J.J. Blech, On Isothermal Squeeze Films, J. Lubr. Technol. 105 (1983) 615. doi:10.1115/1.3254692.

[27] M.M. Altuğ Biçak, M.D. Rao, Analytical modeling of squeeze film damping for rectangular elastic plates using Green's functions, J. Sound Vib. 329 (2010) 46174633. doi:10.1016/j.jsv.2010.05.008.

[28] B. Ahmad, R. Pratap, Analytical evaluation of squeeze film forces in a CMUT with sealed air-filled cavity, IEEE Sens. J. 11 (2011) 2426-2431. doi:10.1109/JSEN.2011.2119397.

[29] I.B. Crandall., The Air-Damped Vibrating System: Theoretical Calibration of the Condenser Transmitter, Phys. Rev. 11 (1918) 449-460. doi:10.1103/PhysRev.11.449.

[30] R.B. Darling, C. Hivick, J. Xu, Compact analytical modeling of squeeze film damping with arbitrary venting conditions using a Green's function approach, Sensors Actuators A Phys. 70 (1998) 32-41. doi:10.1016/S0924-4247(98)00109-5.

[31] S. Jennings, The Mean Free Path in Air, J. Aerosol Sci. 19 (1988) 159-166. doi:10.1063/1.3027060.

[32] T. Veijola, H. Kuisma, J. Lahdenperä, T. Ryhänen, Equivalent-circuit model of the squeezed gas film in a silicon accelerometer, Sensors Actuators A. Phys. 48 (1995) 239-248. doi:10.1016/0924-4247(95)00995-7.

[33] I.O. Wygant, M. Kupnik, B.T. Khuri-Yakub, Analytically calculating membrane displacement and the equivalent circuit model of a circular CMUT cell, in: IEEE Int. Ultrason. Symp. IUS, 2008: pp. 2111-2114. 
(a)

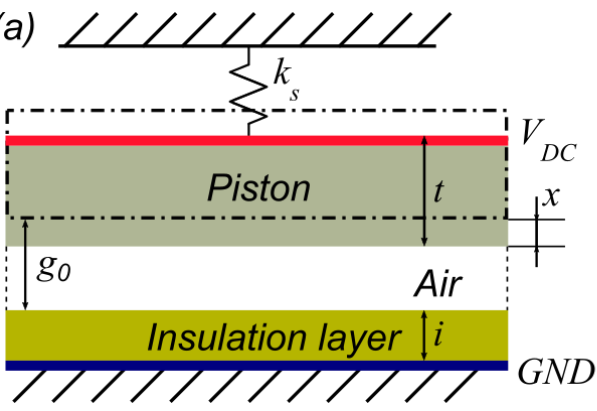

(b)

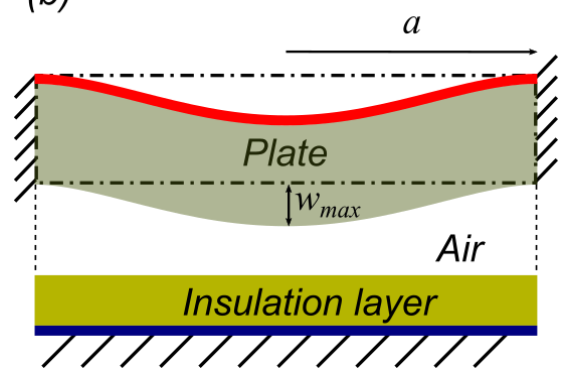

Fig. 1. Cross sectional view of CMUT models with applied bias voltage:

(a) rigid piston transducer; (b) flexible plate transducer.

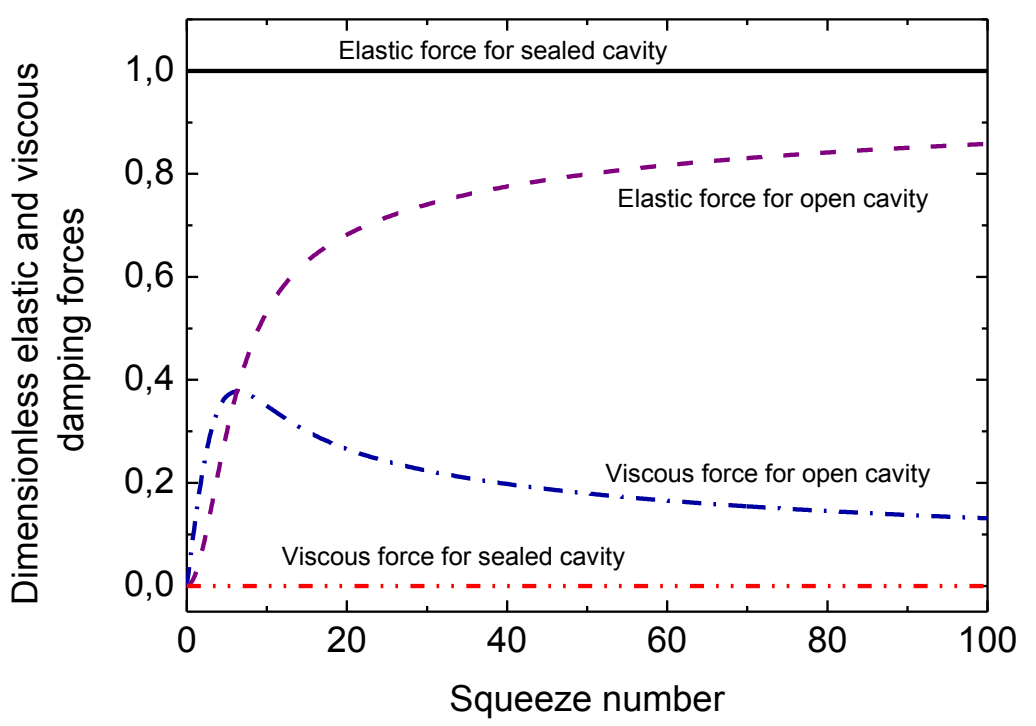

Fig. 2. Dimensionless elastic and viscous damping forces according to the squeeze number for circular piston with open and sealed cavities. 


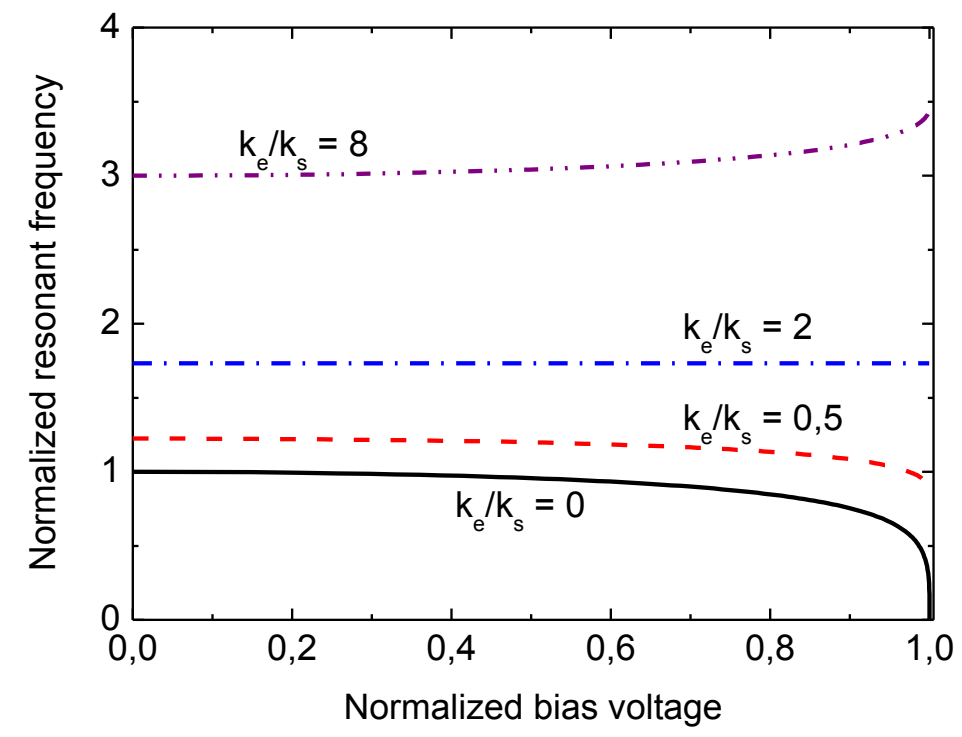

Fig. 3. Normalized resonant frequency as a function of normalized bias voltage for different air spring constant to mechanical spring constant ratio.

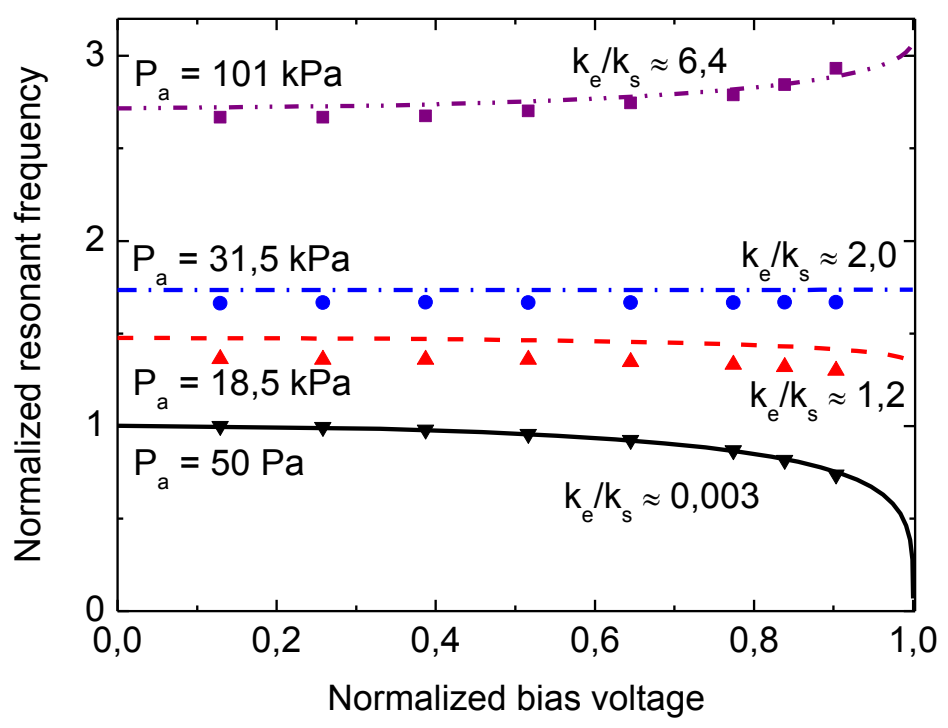

Fig. 4. Normalized resonant frequency according to normalized bias voltage for different pressure for CMUT with $167 \mu \mathrm{m}$ radius (Analytically calculated results: lines, experimental data: dots). 


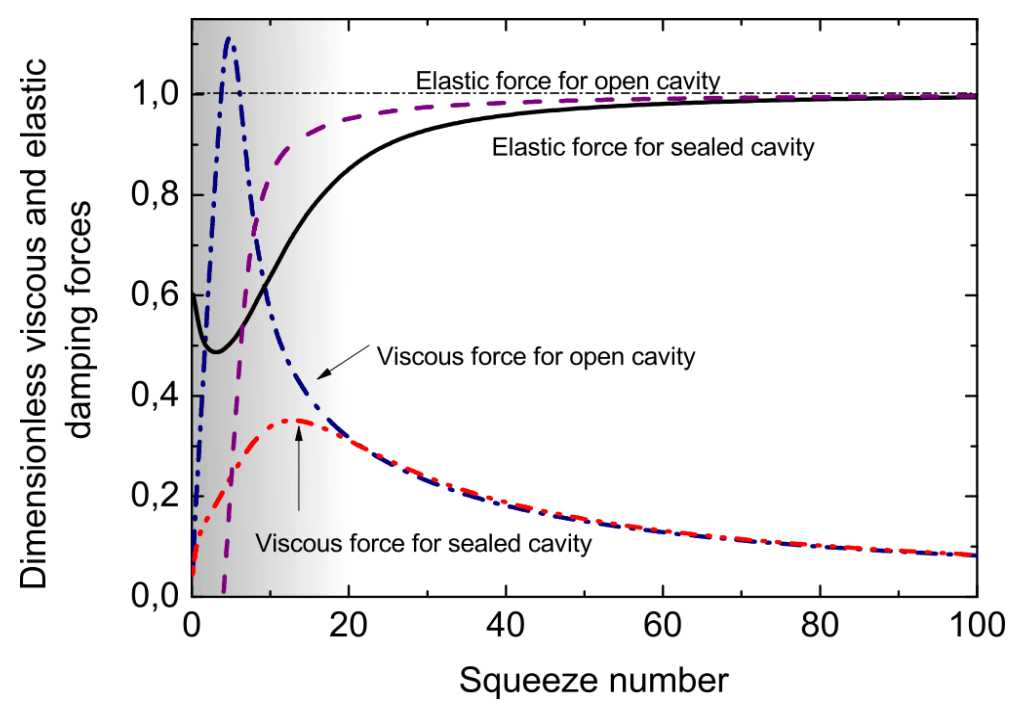

Fig. 5. 2D FEM dimensionless viscous and elastic damping forces according to the squeeze number for open and sealed cavity $(a=150 \mu \mathrm{m}, t=2.3 \mu \mathrm{m})$.

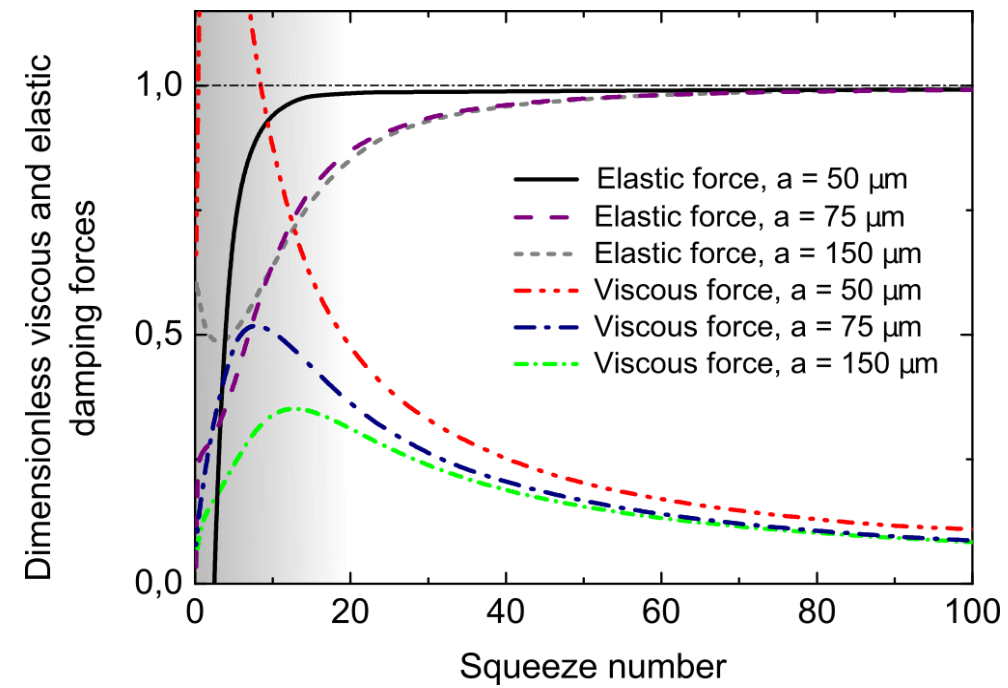

Fig. 6. 2D FEM dimensionless viscous and elastic damping forces according to the squeeze number for different circular CMUT radii with sealed cavity $(t=2.3 \mu \mathrm{m})$.

(a)

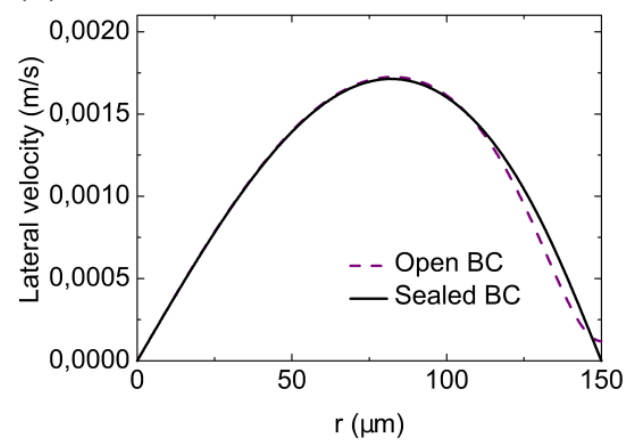

(b)

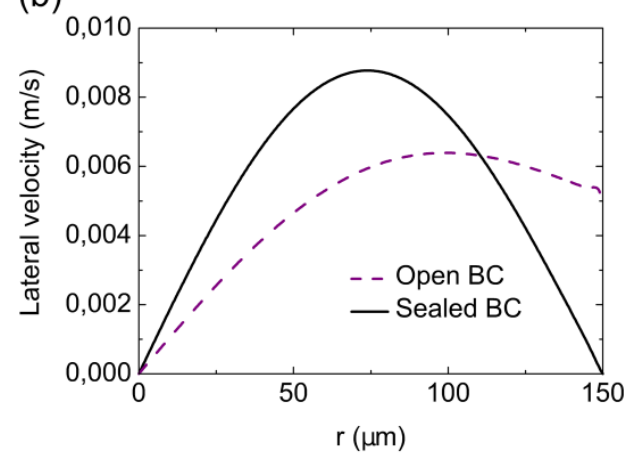

Fig 7. Lateral velocity profile from the center to the periphery of open and sealed cavity in the vicinity of the resonant frequency: (a) $g_{0}=1 \mu \mathrm{m}, a=150 \mu \mathrm{m}$; (b) $g_{0}=10 \mu \mathrm{m}, a=150 \mu \mathrm{m}$. 


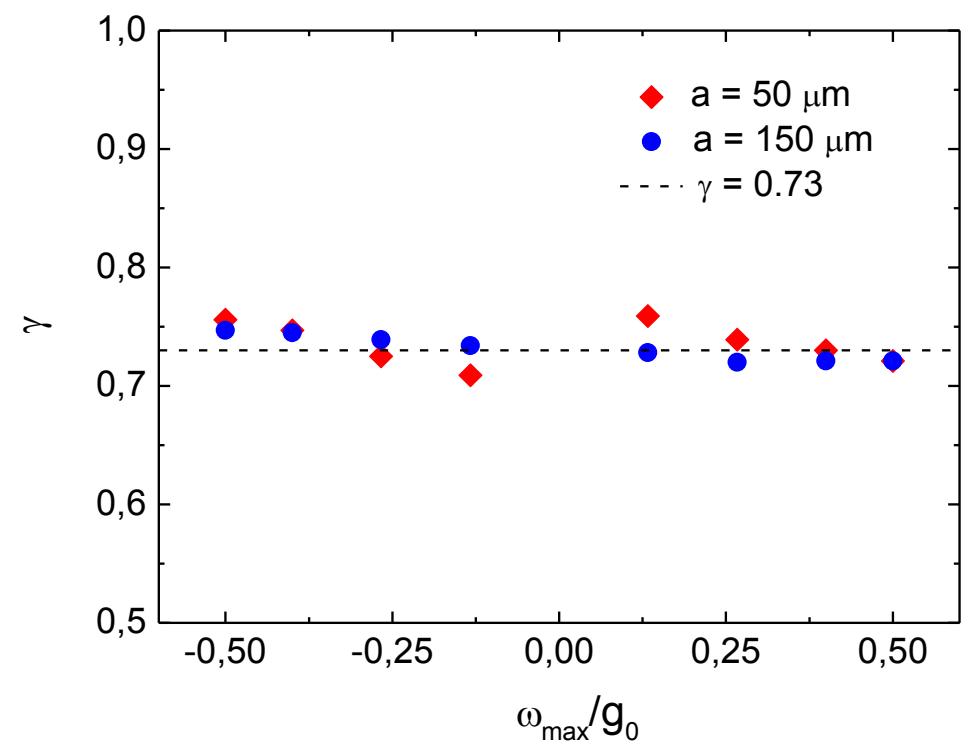

Fig. 8. "Effective" gap constant $(\gamma)$ for different maximum plate displacement

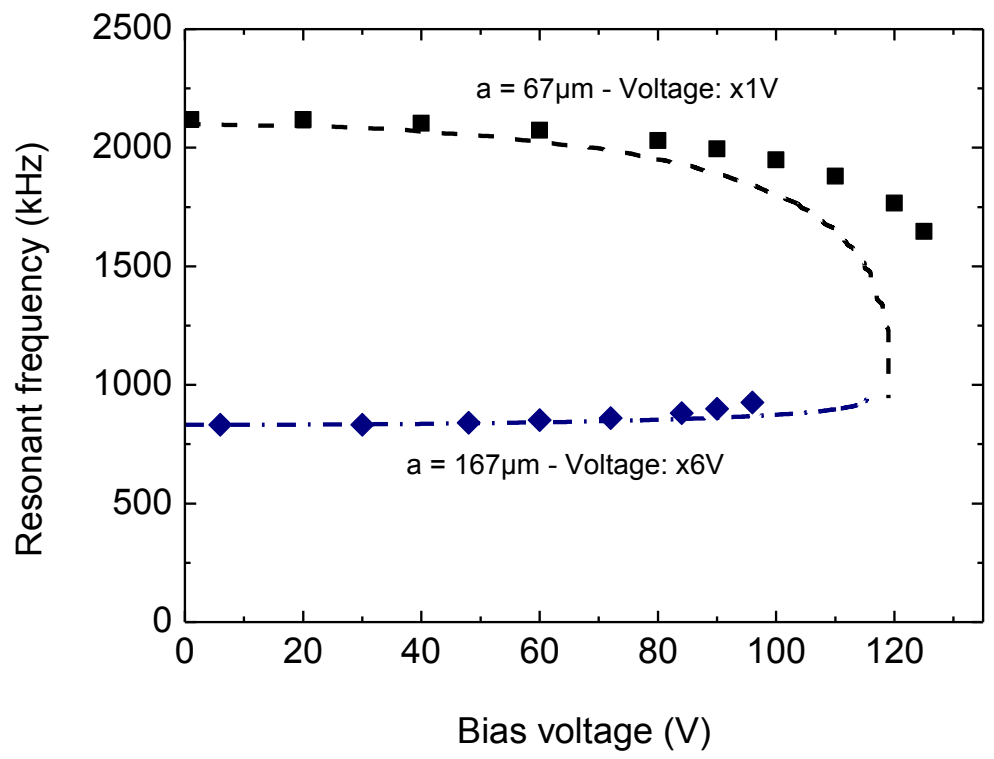

Fig. 9. Resonant frequency according to the DC bias voltage (Analytical air spring constant model: dash/dash dot line, experimental data: dots) 
(a)

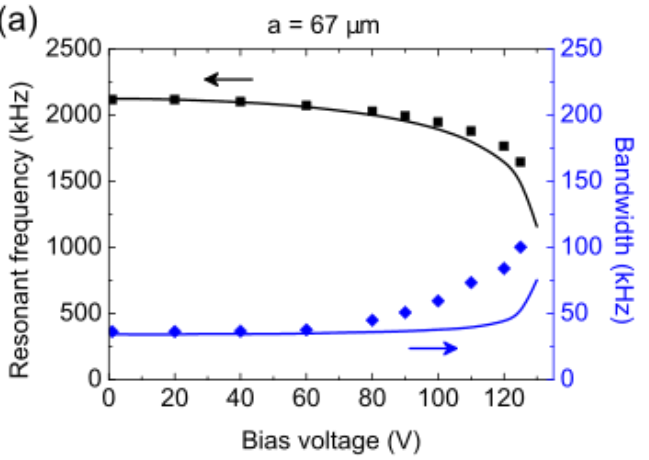

(c)

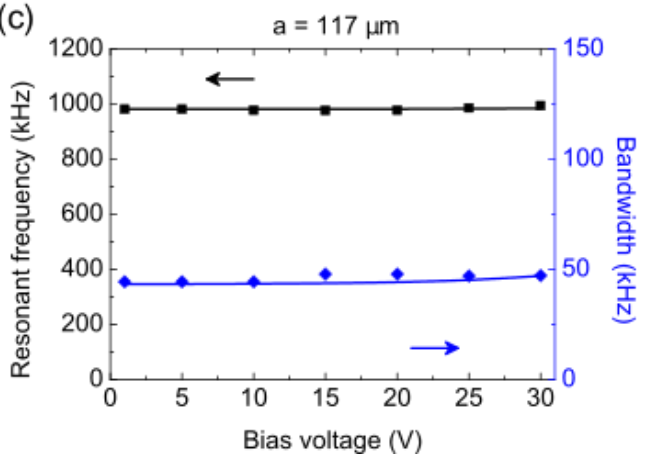

(b)

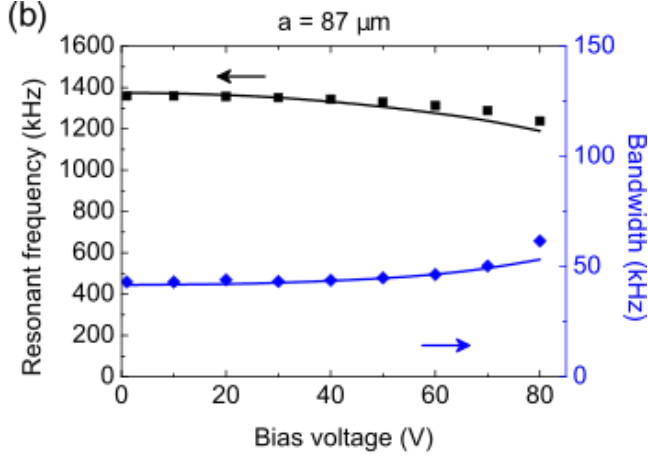

(d)

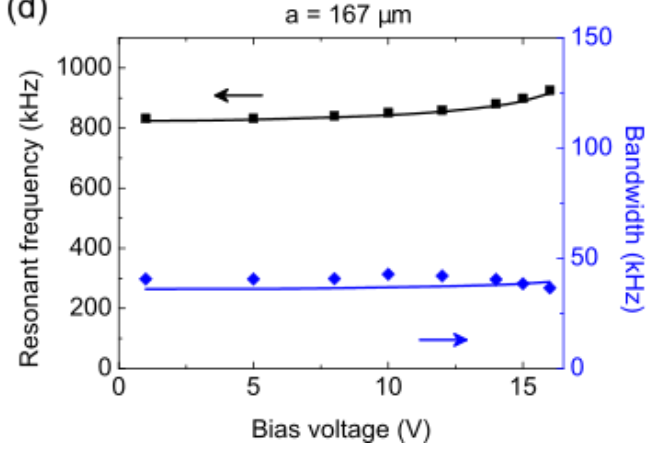

Fig. 10. Resonant frequency and bandwidth according to the DC bias voltage (FEM calculated: solid line, experimental data: dots) 